\title{
Multicenter prospective trial of total gastrectomy versus proximal gastrectomy for upper third cT1 gastric cancer
}

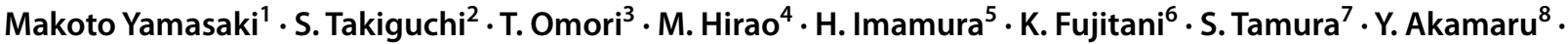

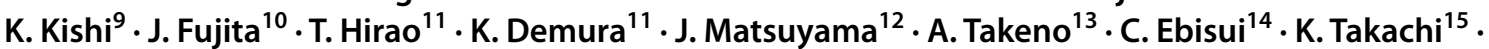 \\ O. Takayama ${ }^{16} \cdot$ H. Fukunaga $^{17} \cdot$ K. Okada $^{18} \cdot$ S. Adachi ${ }^{19}$. S. Fukuda ${ }^{20} \cdot$ N. Matsuura ${ }^{1} \cdot$ T. Saito $^{1}$ - T. Takahashi ${ }^{1}$. \\ Y. Kurokawa ${ }^{1} \cdot$ M. Yano ${ }^{14} \cdot$ H. Eguchi ${ }^{1} \cdot$ Y. Doki $^{1}$
}

Received: 10 July 2020 / Accepted: 28 September 2020 / Published online: 29 October 2020

(c) The International Gastric Cancer Association and The Japanese Gastric Cancer Association 2020

\begin{abstract}
Background The appropriate surgical procedure for patients with upper third early gastric cancer is controversial. We compared total gastrectomy (TG) with proximal gastrectomy (PG) in this patient population.

Methods A multicenter, non-randomized trial was conducted, with patients treated with PG or TG. We compared short- and long-term outcomes between these procedures.

Results Between 2009 and 2014, we enrolled 254 patients from 22 institutions; data from 252 were included in the analysis. These 252 patients were assigned to either the PG $(n=159)$ or TG $(n=93)$ group. Percentage of body weight loss $(\% \mathrm{BWL})$ at 1 year after surgery, i.e., the primary endpoint, in the PG group was significantly less than that of the TG group $(-12.8 \%$ versus $-16.9 \% ; p=0.0001)$. For short-term outcomes, operation time was significantly shorter for PG than TG ( $252 \mathrm{~min}$ versus $303 \mathrm{~min} ; p<0.0001$ ), but there were no group-dependent differences in blood loss and postoperative complications. For long-term outcomes, incidence of reflux esophagitis in the PG group was significantly higher than that of the TG group $(14.5 \%$ versus $5.4 \% ; p=0.02)$, while there were no differences in the incidence of anastomotic stenosis between the two (5.7\% versus $5.4 \% ; p=0.92)$. Overall patient survival rates were similar between the two groups (3-year survival rates: $96 \%$ versus $92 \%$ in the PG and TG groups, respectively; $p=0.49$ ).

Conclusions Patients who underwent PG were better able to control weight loss without worsening the prognosis, relative to those in the TG group. Optimization of a reconstruction method to reduce reflux in PG patients will be important.
\end{abstract}

Keywords Upper third gastric cancer · Total gastrectomy · Proximal gastrectomy

\section{Introduction}

Gastric cancer is one of the most common cancers worldwide, and has a high mortality rate [1,2]. The incidence of cancer localized to the upper third of the stomach and gastroesophageal junction has been increasing in both Western and Eastern countries [3-8]. Patients with upper third gastric cancer undergo surgical treatment in the form of total gastrectomy (TG) or proximal gastrectomy (PG). TG is widely

Electronic supplementary material The online version of this article (https://doi.org/10.1007/s10120-020-01129-6) contains supplementary material, which is available to authorized users.

Makoto Yamasaki

myamasaki@gesurg.med.osaka-u.ac.jp

Extended author information available on the last page of the article used as a standard treatment for these patients [9-11], and while it ensures better tumor margins and a more radical lymphadenectomy, it can lead to postoperative malnutrition. One recent report found that relative to TG, PG was associated with less postoperative weight loss and better nutrition, among other benefits, which were attributed to the preservation of the gastric fundic gland region; no difference was found in postoperative prognosis between TG and PG [12-16]. To date, all studies comparing TG with PG in patients with upper third gastric cancer have been retrospective.

Against this backdrop, we conducted a multicenter prospective non-randomized trial to compare the short-term and long-term efficacies of TG with those of PG for patients with clinically diagnosed $\mathrm{T} 1$ gastric cancer in the upper third of the stomach. We aimed to clarify whether these 
procedures could be used as standard treatment for this patient population.

\section{Methods}

\section{Patients}

Patients from 22 institutions, belonging to the Clinical Study Group of Osaka University upper GI Group, were enrolled in this multicenter non-randomized trial. Each institution was approved as a training institute by the Japan Society of Gastroenterological Surgery. Eligibility was dependent upon a clinical diagnosis of early gastric cancer (cT1) in the upper third of the stomach without involvement of the esophagogastric junction according to the Japan Gastric Cancer Association (JGCA) classification [17], with the possibility of preservation of more than half of the distal stomach in curative resection. Eligibility criteria also included an Eastern Cooperative Oncology Group (ECOG) performance status of 0,1 , or 2 , and no serious vital organ dysfunction (heart, pulmonary, liver, renal, and hematologic). All patients provided written informed consent before enrollment. The study protocol was approved by the institutional review board of each participating hospital. This study was registered with the University Hospital Medical Information Network Clinical Trials Registry (UMIN-CR) of Japan (Identification number: UMIN000003339).

\section{Treatment}

Eligible patients underwent total gastrectomy (TG) or proximal gastrectomy (PG) based on their preference after receiving a thorough explanation of both procedures from the attending surgeon.

All surgeries were performed with curative intent to the extent possible based on the assigned procedure. However, the final extent of gastric resection depended on the intraoperative judgment of the attending surgeons. In general, patients underwent D1+ lymphadenectomy, but underwent D2 lymphadenectomy if attending surgeons intraoperatively determined there was evidence of metastasis to the lymph nodes. Reconstruction methods after gastrectomy were not specified. Gastrectomies were performed either by open laparotomy or laparoscopic surgery. The surgeons with sufficient experience with both procedures, approaches and reconstructions had participated as operator or leading assistants.

\section{Outcomes}

The primary endpoint was the percentage of body weight loss (\%BWL) at 1 year after surgery. Secondary endpoints were \%BWL, serum albumin, hemoglobin, and lymphocyte count during 3 years after surgery, postoperative complications, and overall survival (OS). Postoperative complications were graded according to the Clavien-Dindo classification of surgical complications, with events of grade II or higher considered to be complications [18]. Reflux esophagitis was confirmed by endoscopic examination at 1 year after surgery and assessed using the Los Angeles (LA) classification [19, 20]. Severe esophagitis was defined as grade $C$ or $D$ in the LA classification. Anastomotic stenosis was noted in any patient for whom the dilatation was performed by balloon because the endoscope could not pass through the esophagogastric or esophagojejunal anastomosis.

\section{Statistical analysis}

We planned to enroll 250 patients for the primary analysis. This sample size would provide a power of $80 \%$ and a onesided significance level of 0.05 to detect superiority in terms of \% BWL 1 year after gastrectomy. We anticipated BWL in $7 \%$ of patients in the PG group and $12 \%$ of patients in the TG group, allowing for approximately $10 \%$ missing data for any reason (e.g., lost to follow-up or death).

Continuous numerical data are presented as means and standard deviations (SD), or median, minimum (min) and maximum (max). Student's $t$ test or the Mann-Whitney $U$ test and $\chi^{2}$ test were used to compare continuous and categorical variables, respectively. and the distribution of dichotomous data is presented as percentage and $95 \%$ confidence interval (CI). All $p$ values less than 0.05 were considered statistically significant.

\section{Results}

\section{Patient characteristics}

Between May 2009 and April 2014, 254 patients from 22 institutions were enrolled in the trial. Of these, 164 and 90 patients underwent PG and TG, respectively (Fig. 1). Of the 164 patients enrolled in the PG group, 159 (97\%) completed PG, while four patients switched to TG and one patient received distal gastrectomy. Those who switched to TG from PG did so because positive distal margins were found intraoperatively $(n=2)$, or because the cancer was found to be at an advanced stage and required D2 lymphadenectomy. Of the 90 patients enrolled in the TG group, 89 (99\%) completed TG, while one patient underwent distal gastrectomy. Ultimately, 159 patients underwent PG and 93 underwent $\mathrm{TG}$, and data were analyzed accordingly (PG group versus 


\section{Trial profile}

Enrolled patients with upper third early gastric cancer

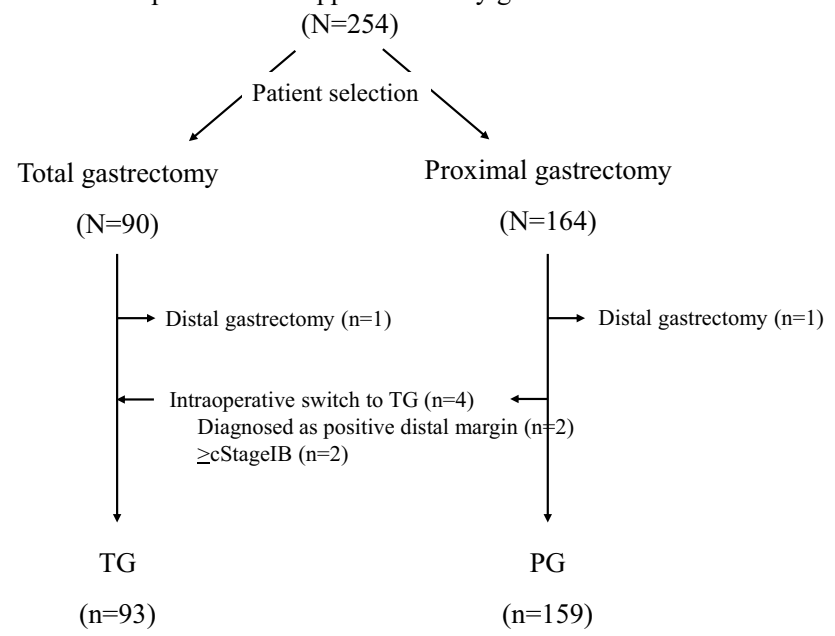

Fig. 1 Trial profile. $T G$ total gastrectomy, $P G$ proximal gastrectomy

Table 1 Patient characteristics

\begin{tabular}{|c|c|c|c|}
\hline & TG $(n=93)$ & PG $(n=159)$ & $p$ \\
\hline Age (years)* & $67.5(41-85)$ & $68.5(32-85)$ & 0.36 \\
\hline \multicolumn{4}{|l|}{ Sex } \\
\hline Male & $67(72 \%)$ & $116(73 \%)$ & \multirow[t]{2}{*}{0.89} \\
\hline Female & $26(28 \%)$ & $43(27 \%)$ & \\
\hline BMI $\left(\mathrm{kg} / \mathrm{m}^{2}\right)^{*}$ & $22.9(14.8-32.2)$ & $23(16.6-32.4)$ & 0.88 \\
\hline \multicolumn{4}{|l|}{ PS } \\
\hline 0 & $76(82 \%)$ & $145(91 \%)$ & \multirow[t]{3}{*}{0.06} \\
\hline 1 & $12(13 \%)$ & $12(8 \%)$ & \\
\hline 2 & $5(5 \%)$ & $2(1 \%)$ & \\
\hline $\mathrm{Hb}(\mathrm{g} / \mathrm{dL})^{*}$ & $13.5(9.3-18.5)$ & $13.3(7.4-16.5)$ & 0.86 \\
\hline $\operatorname{Alb}(\mathrm{g} / \mathrm{dL})^{*}$ & $4.1(3.0-4.8)$ & $4.1(3.0-5.2)$ & 0.86 \\
\hline \multicolumn{4}{|l|}{ cT } \\
\hline T1a & $17(18 \%)$ & $26(16 \%)$ & \multirow[t]{2}{*}{0.69} \\
\hline $\mathrm{T} 1 \mathrm{~b}$ & $76(82 \%)$ & $134(84 \%)$ & \\
\hline \multicolumn{4}{|l|}{$\mathrm{cN}$} \\
\hline 0 & $92(99 \%)$ & $157(99 \%)$ & \multirow[t]{4}{*}{0.89} \\
\hline 1 & $1(1 \%)$ & $2(1 \%)$ & \\
\hline 2 & 0 & 0 & \\
\hline 3 & 0 & 0 & \\
\hline \multicolumn{4}{|l|}{ cStage } \\
\hline IA & $92(99 \%)$ & $157(99 \%)$ & \multirow[t]{4}{*}{0.89} \\
\hline IB & $1(1 \%)$ & $2(1 \%)$ & \\
\hline II & 0 & 0 & \\
\hline III & 0 & 0 & \\
\hline
\end{tabular}

${ }^{*}$ Values are presented as medians (min-max)

TG group). Baseline characteristics were balanced between the two groups (Table 1).

\section{Treatment and complications}

Operative outcomes are summarized in Table 2. In total, 140 patients (31 with TG and 109 with PG) underwent gastrectomy by open laparotomy, a laparoscopic approach taken significantly more often in the TG group relative to the PG group. All patients in the TG group received a Roux-en-Y as the reconstruction procedure. Of the 159 patients in the PG group, esophagogastrostomy anastomosis (EG), jejunal interposition (JI), and double-tract reconstruction (ET) were used as reconstruction procedures in 103, 28, and 28 patients, respectively. Mean operative time was significantly shorter in the PG group than in the TG group (252 min versus $303 \mathrm{~min} ; p<0.0001$ ). Blood loss in the two groups was comparable. The number of retrieved lymph nodes and the extent of lymph node dissection were more and wider in TG than PG, respectively. Pathologically T stage showed significantly more advanced in TG than in PG $(p=0.003)$.

Postoperative complications are shown in Table 3. We found no significant differences between the two groups in any of the observed complications, including postoperative bleeding, anastomotic leakage, pancreatic fistula, and ileus. Overall in-hospital mortality was observed in one patient $(0.4 \%)$ in the TG group who underwent massive enterectomy by strangulation ileus after surgery, which later resulted in short bowel syndrome. This patient died of heart failure on postoperative day 138 . With regard to long-term complications, the incidence of reflux esophagitis was significantly higher in the PG group than in the TG group (14.5\% versus $5.4 \% ; p=0.02$ ), but we observed no group-dependent differences in the rates of severe reflux esophagitis or those with grade $\mathrm{C}$ or D in the LA classification $(2.5 \%$ vs $1.1 \%$ in the PG and TG groups, respectively). We also observed no significant differences in the rates of anastomotic stenosis between the PG and TG groups (5.7\% vs 5.4\%). Forty eight of 159 (30\%) patients with PG, compared with no patient with TG, were prescribed proton pump inhibitor (PPI). A comparison of patients with PPI and those without PPI in the PG group showed that there were no differences in the rate of reflux esophagitis between patients with and without PPI (19\% versus $13 \%$, respectively; $p=0.3$ ).

Rates of reflux esophagitis and anastomotic stenosis according to each reconstruction method within the PG group were also compared. Incidences of reflux esophagitis for EG, DT, and JI were 18.5, 7.1, and 7.1\%, respectively, while those of anastomotic stenosis for EG, DT, and JI were $8.7,0$, and $0 \%$, respectively. The incidence of these complications was significantly higher for EG than for DT and JI.

\section{Postoperative nutritional status}

Postoperative \%BWL by procedure is shown in Fig. 2a. Mean \pm SD \%BWL at 1 year postoperatively was 
Table 2 Surgery and pathologic staging

\begin{tabular}{|c|c|c|c|}
\hline & $\mathrm{TG}(n=93)$ & $\mathrm{PG}(n=159)$ & $p$-value \\
\hline Operative time $(\min )^{*}$ & $296(133-661)$ & $244(110-494)$ & $<0.001$ \\
\hline Blood loss $(\mathrm{mL})^{*}$ & $170(15-1266)$ & $180(10-2379)$ & 0.26 \\
\hline Approach & & & $<0.001$ \\
\hline Open & $33(35 \%)$ & $109(69 \%)$ & \\
\hline Laparoscopic & $60(65 \%)$ & $50(31 \%)$ & \\
\hline Reconstruction & & & - \\
\hline RY & $93(100 \%)$ & 0 & \\
\hline DT & 0 & $28(18 \%)$ & \\
\hline JI & 0 & $28(18 \%)$ & \\
\hline EG & 0 & $103(64 \%)$ & \\
\hline Extent of Lymph node dissection & & & $<0.001$ \\
\hline D1 & 10 & 25 & \\
\hline D1+ & 61 & 134 & \\
\hline D2 & 22 & 0 & \\
\hline Number of retrieved lymph nodes* & $42(1-104)$ & $26(0-76)$ & $<0.001$ \\
\hline Histologic type & & & 0.14 \\
\hline Differentiated & $56(60 \%)$ & $115(72 \%)$ & \\
\hline Undifferentiated & $34(37 \%)$ & $40(25 \%)$ & \\
\hline Other & $3(3 \%)$ & $4(3 \%)$ & \\
\hline pT & & & 0.003 \\
\hline $1 \mathrm{a}$ & $33(35 \%)$ & $39(25 \%)$ & \\
\hline $1 b$ & $38(41 \%)$ & $101(64 \%)$ & \\
\hline 2 & $13(14 \%)$ & $7(4 \%)$ & \\
\hline 3 & $6(6 \%)$ & $10(6 \%)$ & \\
\hline 4 & $3(3 \%)$ & $2(1 \%)$ & \\
\hline $\mathrm{pN}$ & & & 0.65 \\
\hline 0 & $80(86 \%)$ & $142(89 \%)$ & \\
\hline 1 & $7(8 \%)$ & $12(8 \%)$ & \\
\hline 2 & $4(4 \%)$ & $3(2 \%)$ & \\
\hline 3 & $2(2 \%)$ & $2(1 \%)$ & \\
\hline pStage & & & 0.32 \\
\hline IA & $68(73 \%)$ & $131(82 \%)$ & \\
\hline IB & $10(11 \%)$ & $12(8 \%)$ & \\
\hline II & $11(13 \%)$ & $14(9 \%)$ & \\
\hline III & $3(3 \%)$ & $2(1 \%)$ & \\
\hline IV & 0 & 0 & \\
\hline
\end{tabular}

$R Y$ Roux-en-Y, $D T$ double-tract, $J I$ jejunal interposition, $E G$ esophagogastrostomy

*Values are presented as medians (min-max)
$-16.9 \pm 7.9 \%$ in the TG group and $-12.8 \pm 6.7 \%$ in the PG group, with a significant difference between the two groups $(p=0.001)$. These significant differences lasted from 3 months to 3 years after surgery.

Transitions in postoperative serum albumin and hemoglobin levels in the two groups are shown in Fig. 2b, c, respectively. Serum albumin reduction rates did not differ significantly by group at any period during the 3 years after surgery. In addition, no significant changes were noted between the two groups in hemoglobin reduction rates during the first 2 years postoperatively, while hemoglobin reduction rates in the TG group were significantly higher than those in the PG group at 2.5 and 3 years postoperatively. At 1 year postoperatively, 19 of $93(20 \%)$ patients with TG, compared with just 3\% patients with PG, were prescribed vitamin B12 in order to prevent anemia. A comparison of patients with VB12 and those without VB12 in the TG group showed that hemoglobin reduction rates in patients with VB12 were tended, albeit not significantly, to lower than those without VB12 at 1 year postoperatively ( $4.2 \%$ versus $7.2 \%$, respectively; $p=0.07$ ), and were significantly lower than those without VB12 at 
Table 3 Short- and long-term outcomes

\begin{tabular}{|c|c|c|c|}
\hline & $\mathrm{TG}(n=93)$ & $\mathrm{PG}(n=159)$ & $p$-value \\
\hline \multicolumn{4}{|l|}{ Short-term complications } \\
\hline Mortality & $1(1 \%)$ & 0 & 0.19 \\
\hline Total & $20(22 \%)$ & $21(13 \%)$ & 0.09 \\
\hline Bleeding & $1(1 \%)$ & 0 & 0.19 \\
\hline Anastomotic leakage & $8(9 \%)$ & $7(4 \%)$ & 0.17 \\
\hline Ileus & $1(1 \%)$ & $2(1 \%)$ & 0.9 \\
\hline Pancreatic fistula & $3(3 \%)$ & $1(1 \%)$ & 0.11 \\
\hline Hospital stay & $16(6-117)$ & $15(7-88)$ & 0.046 \\
\hline \multicolumn{4}{|l|}{ Long-term complications } \\
\hline Reflux esophagitis & $5(5 \%)$ & $23(14 \%)$ & 0.02 \\
\hline Severe reflux ${ }^{a}$ & $1(1 \%)$ & $4(3 \%)$ & 0.41 \\
\hline Anastomotic stenosis & $5(5 \%)$ & $9(6 \%)$ & 0.92 \\
\hline
\end{tabular}

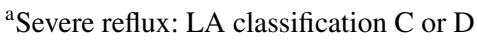

2 years postoperatively (2.2\% versus $7.7 \%$, respectively; $p=0.003)$.

\section{Recurrence and survival}

Eleven (4.4\%) of 252 patients experienced recurrence after surgery. Of these 11 patients, three were in the TG group and eight were in the PG group, with no significant groupdependent difference. Local, lymph node, hematogenous, and dissemination recurrences developed in four (1.6\%), two $(0.8 \%)$, three $(1.2 \%)$, and six (2.4\%) patients, respectively. Local recurrence developed in one (1.1\%) and three (1.9\%) patients in the TG group and PG group, respectively, which did not represent a significant difference between groups $(p=0.61)$. Two cases of lymph node recurrence were noted in the PG group but were localized only to the abdominal para-aortic region. We found no other differences in recurrence type. Overall survival rate in the TG group was similar to that of the PG group (3-year survival rates, 92\% versus 96\%, respectively; $p=0.49$ ) (Fig. 3).

\section{Discussion}

The present study describes the first prospective trial of operative procedures for treating clinically diagnosed cases of early $\mathrm{T} 1$ gastric cancer in the upper third of the stomach. We compared long-term outcomes of PG to those of TG in this patient population.

We initially evaluated postoperative BWL as the primary endpoint because postoperative malnutrition is one of the most common issues experienced by patients undergoing gastrectomy. To date, some retrospective studies have reported that PG resulted in less postoperative BWL than TG [11, 21, 22], consistent with our findings. Given the concurrence of these results, PG may likely become the more commonly performed procedure to avoid postoperative malnutrition for cases of upper third early gastric cancer.

Meanwhile, a few reports have found no differences in postoperative BWL between PG and TG $[12,16]$. An et al. [12] argued that PG was associated with a markedly higher rate of anastomotic stenosis and reflux esophagitis and provided no benefit in terms of postoperative BWL. In their meta-analysis, $\mathrm{Xu}$ et al. reported that incidences of reflux and anastomotic stenosis were significantly higher for PG than TG. However, their subgroup analysis found that these incidences depended on the reconstruction method used in PG [22]. Specifically, they found that PG with EG was more likely to cause reflux and anastomotic stenosis than TG with Roux-en-Y reconstruction, while PG with DT showed an incidence of reflux and anastomotic stenosis similar to that of TG with Roux-en-Y reconstruction [22].

In this study, three reconstruction methods were performed in the PG group: EG, DT, and JI. The incidence of reflux and anastomotic stenosis tended to be, albeit not significantly, higher for EG than for DT or JI, indicating that optimization of the reconstruction method in PG might decrease these long-term complications (Table 4). For the purpose of verifying the optimal reconstruction method in PG, our research group just recently began a new multicenter randomized control trial of esophagogastrostomy (EG) versus double-tract (DT) reconstruction as reconstruction methods for use in PG (UMIN000040918).

We also evaluated postoperative survival rates as oncological outcomes. Overall postoperative survival rates were equal between the PG and TG groups, consistent with previous reports $[11,12,21]$. Although local recurrence is of primary oncological concern in PG, PG was not inferior to TG for local control. TG involves a more radical resection that prevents residual disease at the gastric margin. One metaanalysis indicated that tumor recurrence tended to increase with PG, but no significant difference in survival was found [22]. Yoo et al. [23] analyzed risk factors for local recurrence following PG and suggested avoiding PG for infiltrative or diffuse-type tumors, those greater than $5 \mathrm{~cm}$ in size, and any tumors that demonstrate serosal invasion. In the present study, local recurrence developed in $1.9 \%$ in PG. PG would be also equivalent in local control to TG as far as the eligibility criteria for PG is limited to upper third early gastric cancer.

To determine the setting of the number of patients in this study, we hypothesized that the weight loss rate in 1 year after TG and PG were 12 and 7\% from the retrospective questionnaire survey by medical records of the participating institutes. Only half of those surveyed answered about the body weight in 1 year after surgery, so the result of this survey may have biased our hypothetical weight loss rate. Actually in this study which we investigated the body weight 
Fig. 2 Comparison of nutritional outcomes, body weight (a), serum albumin (b), and hemoglobin (c) between PG and

TG groups. All postoperative data are represented as percent reduction (mean $\pm \mathrm{SE}$ ) over preoperative data. $* p<0.05$, $* * p<0.01$ : significance level. $P G$ solid line, $T G$ dotted line
(\%BWL)

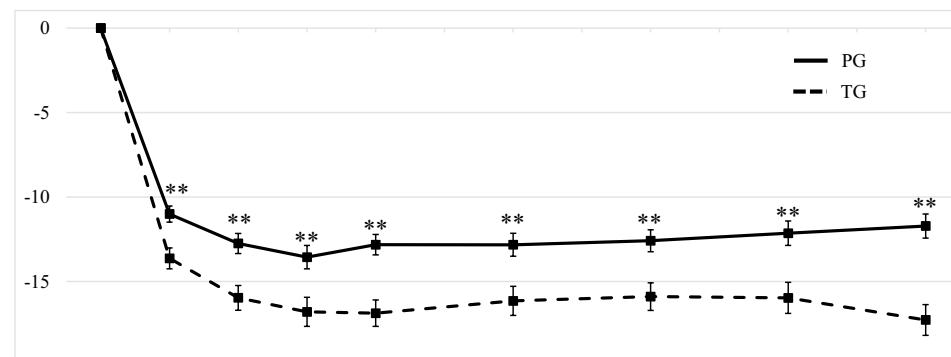

$-20$

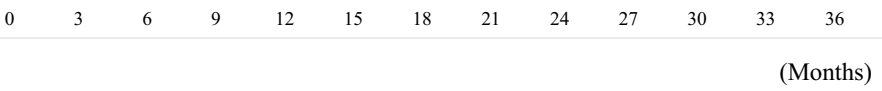

b

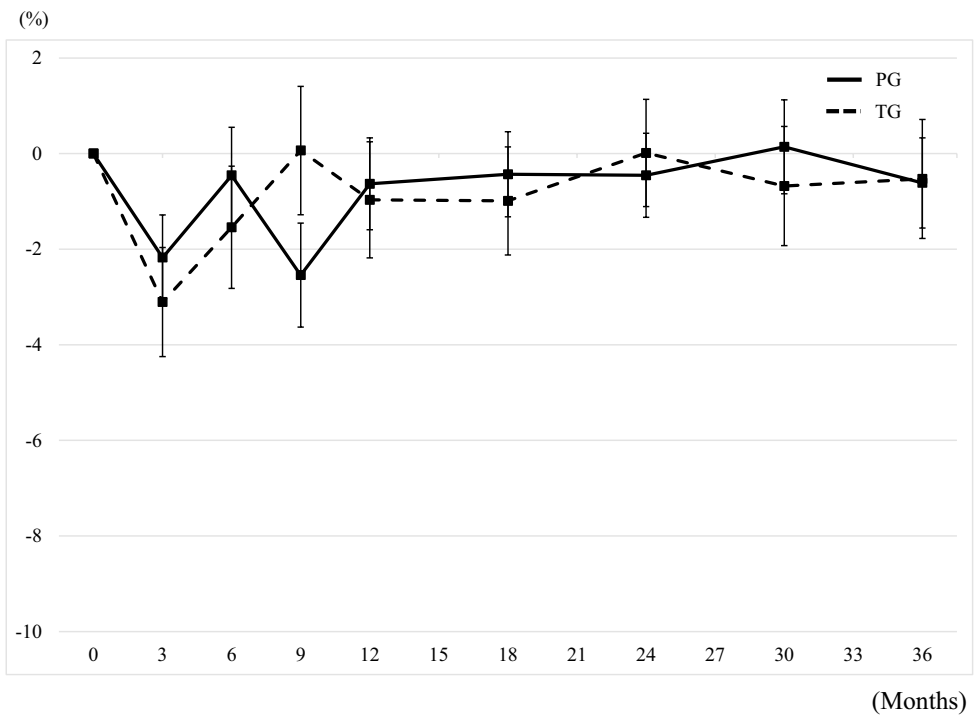

c

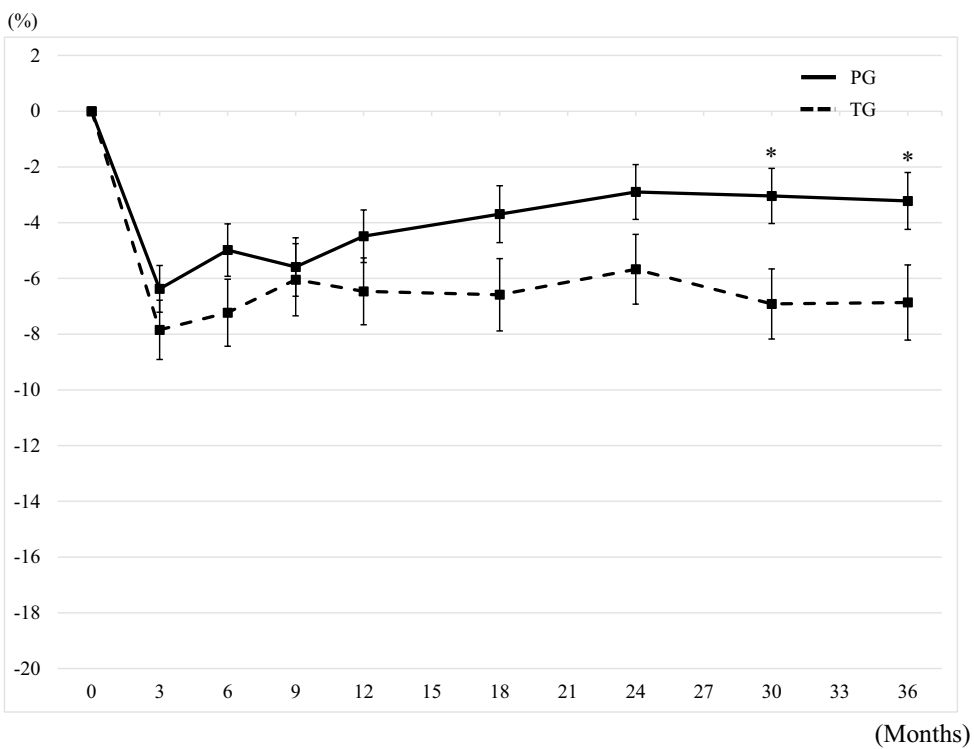




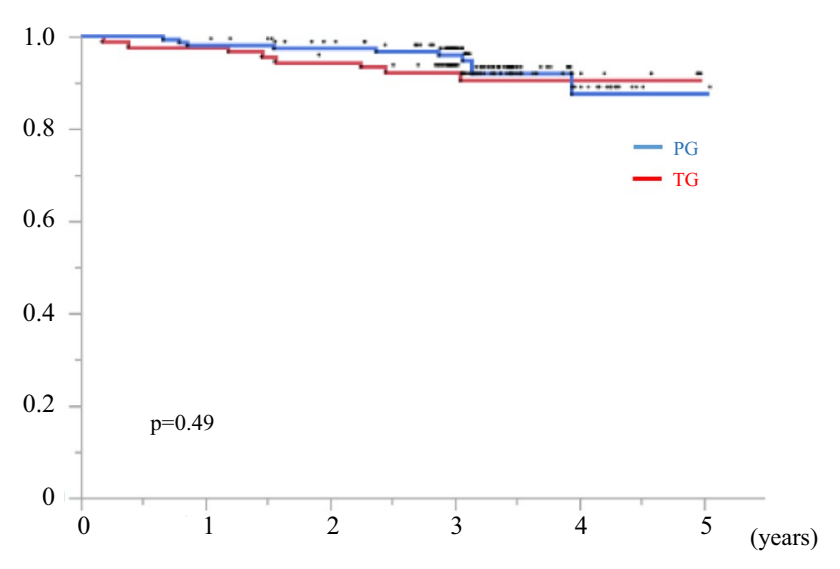

Fig. 3 Survival curves for overall survival after PG and TG. $P G$ blue line, $T G$ red line

for all patients, the weight loss was about $5 \%$ higher than our hypothesis.

One limitation of the present study was that it was not a randomized controlled trial, but rather a non-randomized trial. Specifically, selection of the surgical procedure (PG or TG) and the surgical approach (laparoscopy or open laparotomy) was decided by patients who received an explanation about the procedures from the attending surgeons and provided consent. Thus, there were selection biases.

In fact, five of the 22 participating institutes offered only one procedure (either PG or TG). However, fewer than three patients were enrolled from each of those five institutes. The remaining 17 institutes offered both procedures, which were performed in the remaining $96 \%$ of patients enrolled in this trial.

And TG was performed more frequently by laparoscopic approach compared with PG. Laparoscopic approaches were significantly longer operative time and lower blood loss than Open approach in each operative procedure (TG and PG), but there was no difference in the number of retrieved lymph nodes between both approaches (Supplementary Table 1). Regarding postoperative nutritional status, laparoscopic approach was significantly lower weight loss at 1 year postoperatively than Open approach in TG $(15.7 \pm 7.8 \%$ versus $19.6 \pm 7.7 \%$, respectively; $p=0.04$ ), though there was no difference in weight loss between both approaches in PG (Supplementary Table 1). Preoperative BMI were significantly higher in Open TG than in the other approaches $(24.2 \mathrm{~kg}$ / $\mathrm{m}^{2}$ in Open TG, 22.4 in Lap TG, 23.1 in Open PG and 23.2 in Lap PG). The result of the present study may be due to differences in preoperative BMI.

In conclusion, this study represents the first multicenter prospective non-randomized trial conducted to compare PG and TG as treatment for upper third cT1 gastric cancer. PG was able to reduce weight loss without worsening the prognosis more effectively than TG, suggesting that PG might be the optimal surgical procedure for upper third cT1 gastric cancer. Optimization of the reconstruction method for PG could potentially help alleviate long-term complications.
Table 4 Long-term outcomes by reconstruction method

\begin{tabular}{llllc}
\hline & $\begin{array}{l}\text { TG with RY } \\
n=93\end{array}$ & $\begin{array}{l}\text { PG with JI } \\
n=28\end{array}$ & $\begin{array}{l}\text { PG with DT } \\
n=28\end{array}$ & $\begin{array}{l}\text { PG with EG } \\
n=103\end{array}$ \\
\hline Reflux esophagitis & $5(5.4 \%)$ & $2(7.1 \%)$ & $2(7.1 \%)$ & $19(18.5 \%)$ \\
Severe esophagitis & $1(1.1 \%)$ & $1(3.6 \%)$ & 0 & $3(2.9 \%)$ \\
Anastomotic stenosis & $5(5.4 \%)$ & 0 & 0 & $9(8.7 \%)$ \\
\hline
\end{tabular}

$T G$ total gastrectomy, $R Y$ Roux-en Y, $P G$ proximal gastrectomy, $J I$ jejunal interposition, $D T$ double-tract, $E G$ esophagogastrostomy 
Author contributions All authors contributed to the study conception and design. Material preparation, data collection and analysis were performed by MY, ST, TO, MH, HI, KF, ST, YA, KK, JF, TH, KD, JM, AT, CE, KT, OT, HF, KO, SA, SF, NM, TS, TT, YK, MY, HE and YD. The first draft of the manuscript was written by MY and all authors commented on previous versions of the manuscript. All authors read and approved the final manuscript.

Funding None declared.

\section{Compliance with ethical standards}

Conflict of interest The authors declare that they have no conflict of interest.

Human rights statement All procedures followed were in accordance with the ethical standards of the responsible committee on human experimentation (institutional and national) and with the Helsinki Declaration of 1964 and later versions.

Informed consent Informed consent to be included in the study, or the equivalent, was obtained from all patients.

\section{References}

1. Ferlay J, Soerjomataram I, Dikshit R, et al. Cancer incidence and mortality worldwide: sources, methods and major patterns in GLOBOCAN 2012. Int J Cancer. 2015;136:E359-86.

2. Torre LA, Bray F, Siegel RL, et al. Global cancer statistics, 2012. CA Cancer J Clin. 2015;65:87-108.

3. Dassen AE, Lemmens VE, van de Poll-Franse LV, et al. Trends in incidence, treatment and survival of gastric adenocarcinoma between 1990 and 2007: a population-based study in the Netherlands. Eur J Cancer. 2010;46:1101-10.

4. Steevens J, Botterweck AA, Dirx MJ, et al. Trends in incidence of oesophageal and stomach cancer subtypes in Europe. Eur J Gastroenterol Hepatol. 2010;22:669-78.

5. Wu H, Rusiecki JA, Zhu K, et al. Stomach carcinoma incidence patterns in the United States by histologic type and anatomic site. Cancer Epidemiol Biomark Prev Publ Am Assoc Cancer Res Cospons Am Soc Prev Oncol. 2009;18:1945-52.

6. Yamashita H, Katai H, Morita S, et al. Optimal extent of lymph node dissection for Siewert type II esophagogastric junction carcinoma. Ann Surg. 2011;254:274-80.

7. Crew KD, Neugut AI. Epidemiology of gastric cancer. World J Gastroenterol. 2006;12:354-62.

8. Deans C, Yeo MS, Soe MY, et al. Cancer of the gastric cardia is rising in incidence in an Asian population and is associated with adverse outcome. World J Surg. 2011;35:617-24.
9. Ooki A, Yamashita K, Kikuchi S, et al. Clinical significance of total gastrectomy for proximal gastric cancer. Anticancer Res. 2008;28:2875-83.

10. Katai H, Morita S, Saka M, et al. Long-term outcome after proximal gastrectomy with jejunal interposition for suspected early cancer in the upper third of the stomach. Br J Surg. 2010;97:558-62.

11. Ichikawa D, Komatsu S, Kubota T, et al. Long-term outcomes of patients who underwent limited proximal gastrectomy. Gastric Cancer. 2014;17:141-5.

12. An JY, Youn HG, Choi MG, et al. The difficult choice between total and proximal gastrectomy in proximal early gastric cancer. Am J Surg. 2008;196:587-91.

13. Huh YJ, Lee HJ, Oh SY, et al. Clinical outcome of modified laparoscopy-assisted proximal gastrectomy compared to conventional proximal gastrectomy or total gastrectomy for upper-third early gastric cancer with special references to postoperative reflux esophagitis. J Gastric Cancer. 2015;15:191-200.

14. Ikeguchi M, Kader A, Takaya S, et al. Prognosis of patients with gastric cancer who underwent proximal gastrectomy. Int Surg. 2012;97:275-9.

15. Kondoh Y, Okamoto Y, Morita M, et al. Clinical outcome of proximal gastrectomy in patients with early gastric cancer in the upper third of the stomach. Tokai J Exp Clin Med. 2007;32:48-53.

16. Shiraishi N, Adachi Y, Kitano S, et al. Clinical outcome of proximal versus total gastrectomy for proximal gastric cancer. World $\mathbf{J}$ Surg. 2002;26:1150-4.

17. Japanese Gastric Cancer Association. Japanese classification of gastric carcinoma: 3rd English edition. Gastric Cancer. 2011;14:101-12.

18. Dindo D, Demartines N, Clavien PA. Classification of surgical complications: a new proposal with evaluation in a cohort of 6336 patients and results of a survey. Ann Surg. 2004;240:205-13.

19. Armstrong D, Bennett JR, Blum AL, et al. The endoscopic assessment of esophagitis: a progress report on observer agreement. Gastroenterology. 1996;196(111):85-92.

20. Visick AH. Measured radical gastrectomy; review of 505 operations for peptic ulcer. Lancet. 1948;1:551-5.

21. Ushimaru Y, Fujiwara Y, Shishido Y, et al. Clinical outcomes of gastric cancer patients who underwent proximal or total gastrectomy: a propensity score-matched analysis. World J Surg. 2018;42:1477-84.

22. Xu Y, Tan Y, Wang Y, et al. Proximal versus total gastrectomy for proximal early gastric cancer. A systematic review and metaanalysis. Medicine (Baltimore). 2019;98:e15663.

23. Yoo CH, Sohn BH, Han WK, Pae WK. Long-term results of proximal and total gastrectomy for adenocarcinoma of the upper third of the stomach. Cancer Res Treat. 2004;36:50-5.

Publisher's Note Springer Nature remains neutral with regard to jurisdictional claims in published maps and institutional affiliations. 


\section{Affiliations}

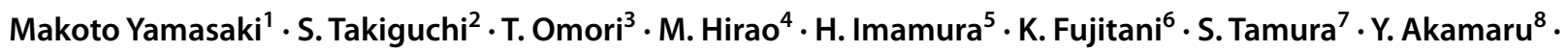

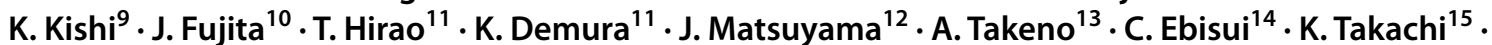

O. Takayama ${ }^{16} \cdot$ H. Fukunaga ${ }^{17} \cdot \mathrm{K}_{\text {. Okada }}{ }^{18} \cdot \mathrm{S}$. Adachi $^{19} \cdot \mathrm{S}_{\text {. Fukuda }}{ }^{20} \cdot$ N. Matsuura ${ }^{1} \cdot$ T. Saito $^{1} \cdot$ T. Takahashi $^{1}$. Y. Kurokawa ${ }^{1} \cdot$ M. Yano ${ }^{14} \cdot$ H. Eguchi ${ }^{1} \cdot$ Y. Doki $^{1}$

1 Department of Gastroenterological Surgery, Graduate School of Medicine, Osaka University, 2-2-E2, Yamadaoka, Suita, Osaka 565-0879, Japan

2 Department of Gastroenterological Surgery, Nagoya City University Graduate School of Medical Sciences, Nagoya, Japan

3 Department of Surgery, Osaka International Cancer Institute, Osaka, Japan

4 Department of Surgery, National Hospital Organization, Osaka National Hospital, Osaka, Japan

5 Department of Surgery, Toyonaka Municipal Hospital, Osaka, Japan

6 Department of Surgery, Osaka General Medical Center, Osaka, Japan

7 Department of Surgery, Yao Municipal Hospital, Osaka, Japan

8 Department of Surgery, Ikeda Municipal Hospital, Osaka, Japan

9 Department of Surgery, Osaka Police Hospital, Osaka, Japan

10 Department of Surgery, Sakai City Medical Center, Osaka, Japan
11 Department of Surgery, Japan Community Health Care Organization Osaka Hospital, Osaka, Japan

12 Department of Surgery, Higashiosaka City Medical Center, Osaka, Japan

13 Department of Surgery, Kansai Rosai Hospital, Hyogo, Japan

14 Department of Surgery, Suita Municipal Hospital, Osaka, Japan

15 Department of Surgery, Kinki Central Hospital, Hyogo, Japan

16 Department of Surgery, Saiseikai Senri Hospital, Osaka, Japan

17 Department of Surgery, Itami Municipal Hospital, Osaka, Japan

18 Department of Surgery, Hyogo Prefectural Nishinomiya Hospital, Hyogo, Japan

19 Department of Surgery, Nishinomiya Municipal Central Hospital, Hyogo, Japan

20 Department of Surgery, Kindai University Nara Hospital, Osaka, Japan 